\title{
OTIMIZAÇÃO DA ALOCAÇÃO DE RECURSOS EM PORTFÓLIO DE PROJETOS - ESTUDO DE CASO DO SISTEMA DE OBRAS DO EXÉRCITO BRASILEIRO
}

\author{
Marion Gomes de Moraes Fernandes \\ Instituto Militar de Engenharia \\ Praça General Tibúrcio nº 80 - Rio de Janeiro - RJ \\ eng.marionfernandes@gmail.com \\ Orivalde Soares da Silva Júnior \\ Instituto Militar de Engenharia \\ Praça General Tibúrcio nº 80 - Rio de Janeiro - RJ \\ orivalde@ime.eb.br
}

\section{RESUMO}

Este trabalho tem por objetivo abordar alguns conceitos de gerenciamento de portfólio de projetos, análise multicritério e programação linear com o intuito de aplicação em um estudo de caso. Utiliza-se da ferramenta de decisão multicritério Structured PairWise Comparison - SPC, também conhecido como Analytic Hierarchy Process - AHP simplificado, para o cálculo de pesos para os projetos de um portfólio. A partir de dados reais do Sistema de Saúde do Exército Brasileiro e de alguns dados fictícios, aplica-se ferramentas de programação linear para otimizar a alocação de recursos aos projetos. Tal metodologia pode colaborar com a priorização de projetos e alocação de recursos do Sistema de Obras do Exército Brasileiro executado pela Diretoria de Obras Militares.

Palavra-chave: Otimização; Gerenciamento; Portfólio; Projetos; Decisão Multicritério.

\section{ABSTRACT}

This work aims to discourse about some concepts of project portfolio management, multicriteria analysis and linear programming with the intention of applying it in a case study. It uses the multicriteria decision-making tool Structured Pair -Wise Comparison SPC, also known as simplified Analytic Hierarchy Process - AHP, to calculate weights for portfolio projects. Based on actual data from the Brazilian Army Health System and some fictitious data, linear programming tools are applied to optimize the allocation of resources to projects. Such methodology can collaborate with the prioritization of projects and allocation of resources of the System of Works of the Brazilian Army executed by the Directory of Military Works.

Keywords: Optimization; Management; Portfolio; Projects; Multicriteria Decision.

\section{Como Citar:}

FERNANDES, Marion Gomes de Moraes; SILVA JÚNIOR, Orivalde Soares da. Otimização da alocação de recursos em portfólio de projetos - estudo de caso do Sistema de Obras do Exército Brasileiro. In: SIMPÓSIO DE PESQUISA OPERACIONAL E LOGÍSTICA DA MARINHA, 19., 2019, Rio de Janeiro, RJ. Anais [...]. Rio de Janeiro: Centro de Análises de Sistemas Navais, 2019. 


\section{INTRODUÇÃO}

A limitação de recursos encontrada em grande parte dos órgãos públicos eleva a necessidade contínua e crescente de melhoria dos resultados obtidos nos projetos desenvolvidos. Tal fato gera uma preocupação em tomar decisões corretas no que tange a escolha dos projetos a serem desenvolvidos, assim como no valor investido em cada um deles.

Contudo, a maior parte das decisões de gerência sobre a escolha de projetos baseia-se no bom-senso, experiência e intuição dos gestores Campos (2014). No que se trata de órgãos públicos, a responsabilidade de tomar boas decisões e investir o recurso corretamente ganha proporções ainda maiores do que nas empresas privadas.

Nem todos os órgãos públicos utilizam de métodos decisórios baseados em análises e comparações sistemáticas dos seus projetos, porém tem sido possível observar um movimento positivo no sentido do aprimoramento de instituições e diversas empresas (PMI, 2013).

Diante do exposto, a motivação desta pesquisa é propor uma metodologia que alinhe os objetivos estratégicos da Diretoria de Obras Militares do Exército Brasileiro (DOM) à escolha de projetos de obras de construção e reformas de instalações e colabore com definição dos recursos alocados para cada obra. Serão utilizadas ferramentas de decisão multicritério e ferramentas de Programação Linear. Serão consideradas restrições quanto ao recurso disponível, tempo de aplicação do recurso e proporção de distribuição entre Regiões Militares.

A pesquisa é classificada como qualitativa no que diz respeito a abordagem do problema pois pretende-se demonstrar a estrutura básica de unidades militares da DOM e seus planos estratégicos. Quanto aos objetivos, esta pesquisa é explicativa, pois procura identificar fatores que contribuem na prática de gerenciamento de portfólio e aplicá-los em uma metodologia de apoio à decisão baseada em programação linear.

Esta pesquisa está dividida em quatro seções. Esta introdução é a primeira seção, a segunda seção apresenta o referencial teórico sobre o Método de Análise Hierárquica (AHP) e Programação Linear (PL). A terceira seção apresenta o problema de alocação de recursos da DOM, e a quarta seção descreve o problema matemático, modelagem e aplicação da metodologia proposta em um estudo de caso com dados reais e hipotéticos.

\section{REFERENCIAL TEÓRICO}

\subsection{Gerenciamento de Portfólio}

Segundo o PMBOK - Project Management Body of Knowledge (2008), um projeto é um esforço temporário empreendido para criar um produto, serviço ou resultado exclusivo. O MGP-SISP (BRASIL, 2011) diz que um programa é um conjunto de projetos relacionados, gerenciados de modo coordenado, a fim de obter benefícios não disponíveis se gerenciados individualmente.

A gestão de portfólio de projetos é uma atividade que deve estar alinhada com os objetivos e as estratégias da organização, sem exceder os recursos disponíveis ou violar quaisquer outras restrições de modo a selecionar ou priorizar a carteira de projetos ideal para a empresa (Archer \& Ghasemzadeh, 1999).

Cresce a quantidade de gestores preocupados em obter conhecimento e desenvolver metodologias para fundamentar suas decisões e maximizar o retorno dos seus portfólios. $\mathrm{O}$ portfólio de projetos é, ainda segundo o PMI (2013), a coleção de projetos, programas e outros trabalhos que são agrupados para facilitar o gerenciamento efetivo do esforço para atingir as metas estratégicas do negócio.

Conforme o PMI (2013), “os componentes que formam um portfólio de projetos de uma organização, são quantificáveis e podem ser medidos, ranqueados e priorizados. Desta forma, é possível ser feita uma avaliação fundamentada do alinhamento dos projetos com os objetivos estratégicos da instituição, e do seu desempenho. O objetivo principal do gerenciamento do portfólio de projetos é fazer com que a organização aplique seus recursos nos projetos que trarão maior retorno de forma otimizada”. 


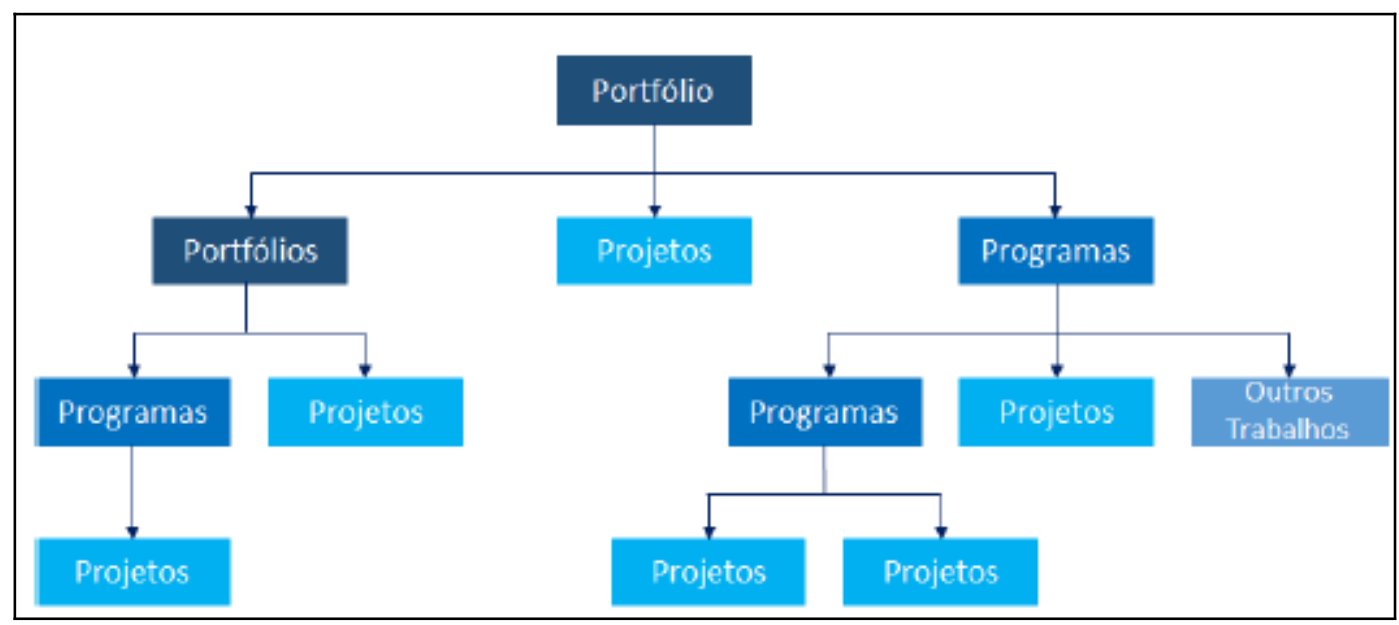

Figura 1: Projetos, programas, portfólios e suas relações

Fonte: Adaptado de PMI (2006)

Ainda segundo o PMI (2013), o gerenciamento do portfólio de projetos, ou gerenciamento do portfólio, é o gerenciamento coordenado dos componentes para atingir os objetivos organizacionais.

O gerenciamento de portfólio inclui processos para coletar, identificar, classificar, avaliar, selecionar, priorizar, equilibrar, e autorizar componentes de revisão dentro do portfólio para avaliar como eles estão em relação aos principais indicadores e o plano estratégico da organização (PMI, 2006).

Para Catelli (2001), “a gestão de portfólio está ligada ao planejamento estratégico da instituição, uma vez que o planejamento estratégico tem como princípio assegurar o cumprimento da missão da empresa, gerando um conjunto de diretrizes estratégicas de caráter qualitativo, que visa orientar a etapa de planejamento operacional”.

Conforme Liesiö, Mild e Salo (2008), usualmente as condições de gerenciamento de um portfólio de projetos não são as adequadas, uma vez que diversos fatores e falta de dados atrapalham a análise. A tomada de decisão geralmente é prejudicada devido a grande quantidade de projetos do portfólio, interdependência entre os projetos, projetos excludentes entre si, muitos critérios a serem atendidos, riscos e informações desconhecidos.

\subsection{MÉtodo de ANÁlise Hierárquica (AHP)}

Segundo Gomes (2007), o método de análise hierárquica é um dos métodos multiatributo mais utilizados e difundidos no mercado mundial. Pode-se explicar tal fato por dois motivos prováveis. O primeiro motivo é por ser um método pioneiro desenvolvido em meados da década de 1970 pelo pesquisador americano Thomas L. Saaty, o qual buscou propor uma abordagem de hierarquizar decisões sob a ótica de múltiplos critérios simultâneos (Saaty, 2006). O segundo motivo é a sua aplicação intuitiva e de fácil entendimento. Quem entende o funcionamento do método, rapidamente o aplica ao estruturar os critérios, definir valores e escolhendo as alternativas.

O método é dividido em três etapas, a primeira etapa é a construção da hierarquia do problema. A segunda etapa e definir prioridades e a terceira é a verificação da consistência. A Figura 2 mostra uma estrutura hierárquica com meta, critérios e relação entre alternativas. 


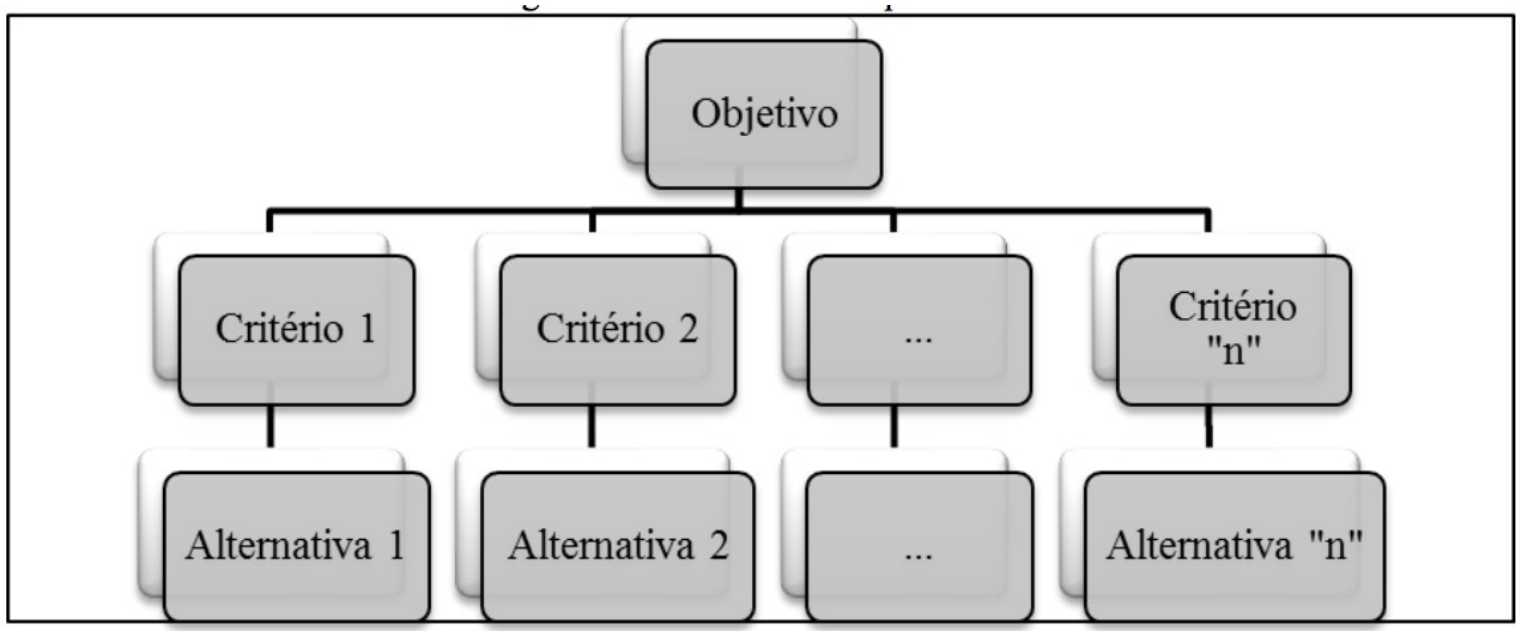

Figura 2: Hierarquia AHP

Fonte: Fusco, (2013)

\subsection{ATRIBUIÇÃO DE VALORES PARA OS CRITÉRIOS}

Uma vez definida a estrutura de hierarquia para o problema de decisão a próxima etapa é determinar os valores relativos para cada critério escolhido. O objetivo desta etapa é verificar a importância de um critério em relação a outro no contexto do problema. Para fazer esta verificação fazem-se comparações dois a dois entre os critérios, chamadas de análises paritárias. Monta-se então uma matriz quadrada chamada de matriz de comparações paritárias, conforme mostra um exemplo na Tabela 1.

Tabela 1: Matriz de Comparações Paritárias

\begin{tabular}{c|cccc}
\hline Critérios & Critério & Critério & .. & Critério \\
& $\mathbf{1}$ & $\mathbf{2}$ & $\mathbf{.}$ & $\boldsymbol{m}$ \\
\hline Critério 1 & 1 & $\mathrm{a}_{12}$ & $\ldots$ & $\mathrm{a}_{1 \mathrm{~m}}$ \\
Critério 2 & $\mathrm{a}_{21}$ & 1 & $\ldots$ & $\ldots$ \\
$\ldots$ & $\ldots$ & $\ldots$ & 1 & $\ldots$ \\
Critério & $\mathrm{a}_{\mathrm{m} 1}$ & $\ldots$ & $\ldots$ & 1 \\
$m$ & & & & \\
\hline
\end{tabular}

O preenchimento desta matriz é feito usando a escala fundamental de Saaty. Esta escala faz a relação entre valores de importância definidos por pessoas e valores numéricos entre 1 e 9. Ou seja, ela traduz o sentimento humano sobre a importância de um critério em relação a outro em números. A Escala Fundamental de Saaty está ilustrada na Tabela 2.

Tabela 2: Escala Fundamental de Saaty

\begin{tabular}{c|c}
\hline $\begin{array}{c}\text { Escala } \\
\text { numérica }\end{array}$ & Escala verbal \\
\hline 1 & Mesma importância \\
3 & Importância moderada de um sobre o outro \\
5 & Importância essencial ou forte \\
7 & Importância muito forte \\
9 & Importância extrema \\
$2,4,6,8$ & Valores intermediários \\
\hline
\end{tabular}


A matriz de comparações paritárias preenchidas conforme a escala fundamental de Saaty (2006) apresenta uma simetria em relação à sua diagonal principal. Isso ocorre, pois o valor dado a um determinado elemento $a_{i j}$ reflete que a importância do critério $i$ é $a_{i j}$ vezes mais importante que o critério $j$. O que faz com que $a_{i j}=1 / a_{j i}$, levando a simetria da matriz.

Depois de preenchida a matriz de comparações paritárias calcula-se o vetor de pesos associado a essa matriz. Este vetor de pesos mostra importância relativa de cada critério em relação aos outros critérios. Tal vetor pode ser obtido de diversas formas, por exemplo, calculando-se a média aritmética de cada linha da matriz, montando um vetor intermediário. Normaliza-se este vetor intermediário, dividindo cada elemento pela soma de todos os seus elementos.

\subsection{ANÁLISE DE INCONSISTÊNCIA}

Durante o preenchimento de cada matriz de comparações paritárias é possível que ocorra algum erro nos julgamentos de valor, chamado de inconsistência. É obrigatório que algumas relações de preferência sejam respeitadas entre os critérios. Considerando os critérios 1,2 e 3, a relação não é inconsistente se ocorrer que se o critério 1 é preferível ao critério 2, e o critério 2 é preferível ao critério 3, então o critério 1 é preferível ao critério 3.

Outra relação de inconsistência trata da intensidade em que um critério é preferível em relação a outro. A inconsistência é determinada por um índice medido com procedimentos específicos e Saaty (2006) definiu que o valor de 0,1 é aceitável para o índice de inconsistência.

\subsection{STRUCTURED PAIR-Wise COMPARISON - SPC}

O método Structured Pair-wise Comparison - SPC baseia-se no método AHP, contudo apresenta uma metodologia mais simplificada, e por este motivo é também conhecido como método AHP simplificado. Segundo Silva e Silva (2009) este método torna o preenchimento da matriz pelo avaliador mais fácil. Outra vantagem é evitar que os critérios mais importantes sejam comparados com os critérios menos importantes. A sequência desta metodologia pode ser apresentada em dois passos:

Passo 1: Apresenta-se os critérios ao avaliador e este os coloca em ordem de prioridade, do mais importante ao menos importante.

Passo 2: Faz a comparação da importância entre os critérios adjacentes na lista ordenada, atribuindo os valores de importância conforme a escala de Saaty.

Passo 3: Calcula-se o vetor de pesos dos critérios.

Passo 4: Cria-se escalas para os critérios e calcula-se a os pesos dos projetos.

Depois de executadas as etapas iniciais descritas, prossegue-se com os procedimentos do método AHP convencional já mencionado, sem a análise de inconsistência.

\subsection{ProgramaÇão Linear}

A Programação Linear (PL) é uma das diversas linhas de estudo pertencentes à área da Pesquisa Operacional (PO). Trata-se de aplicação de ferramentas para solucionar problemas de otimização com vasta aplicação em apoio à decisão. A partir de uma função objetivo, determinam-se restrições que assumem características lineares e chega-se a valores otimizados das variáveis de acordo com os critérios definidos. Devido a estas características, a PL tem tido grande aplicação em problemas gerenciais.

Segundo Oliveira e Ferreira (2011), a Pesquisa Operacional teve início na segunda Guerra Mundial, quando havia necessidade de distribuição dos poucos recursos de forma eficiente dentre as variadas operações militares. Ao longo do tempo, dois fatores foram fundamentais para o crescimento da PO, o avanço das técnicas de formulação de problemas e aumento do uso dos computadores, conforme Moreira (2010). A PO permite criar modelos 
matemáticos e definir algoritmos de solução que mostre como retirar ou resolver uma certa dificuldade (Goldbarg \& Luna, 2005).

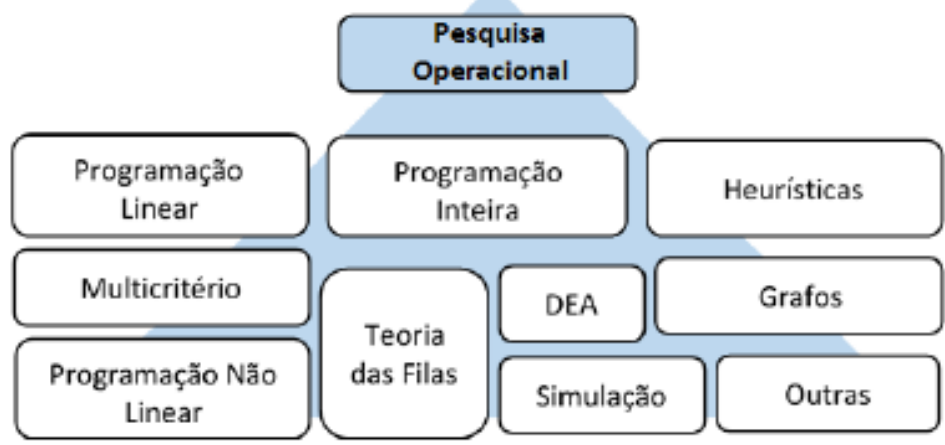

Figura 3: Ferramentas típicas de PO

Fonte: Oliveira e Ferreira (2011)

Para um modelo ser classificado como de programação linear ele deve atender as condições de proporcionalidade, não negatividade, aditividade e separabilidade. A proporcionalidade ocorre quando a quantidade de recursos consumidos por uma dada atividade deve ser proporcional ao nível dessa atividade na solução final do problema. E o custo de cada atividade é proporcional ao nível de operação da atividade. A não negatividade impõe que deve ser sempre possível desenvolver dada atividade em qualquer nível não negativo e qualquer proporção de um dado recurso deve sempre ser utilizado. A aditividade diz que o custo total é a soma das parcelas associadas a cada atividade, e a separabilidade prega que se pode identificar de forma separada o custo (ou consumo de recursos) específico das operações de cada atividade.

\section{METODOLOGIA}

\subsection{Cálculo dos Pesos Pelo Método Structured Pair-Wise CoMparison - SPC}

Para a aplicação deste método foram considerados os seguintes indicadores:

- Custo da obra: refere-se ao valor monetário associado à escolha deste projeto. Quanto de fato à execução desta obra vai custar para a instituição.

- Urgência da obra: reflete quanto tempo à instituição pode esperar até que esta obra fique pronta e em condições de uso, de forma inversa. Ou seja, quanto maior a urgência, menor o tempo disponível de espera. Aqui deve ser considerado que quanto menor o valor alocado para a obra, mais tempo ela vai demorar em ficar pronta.

- Retorno da obra: mede quanto à obra é importante e quanto ela pode melhorar o cumprimento dos objetivos da instituição. A avaliação de retorno foi feita pela DOM, e aqui foi pontuada conforme a escala de retorno feita pelo autor.

Foram criadas escalas para cada um dos critérios e aos projetos foram atribuídos os valores de cada critério seguindo as escalas. A matriz de valores foi normalizada e cada coluna de projeto foi multiplicada pelo vetor de pesos dos critérios. Obtiveram-se então os pesos dos projetos.

Tabela 3: Escala dos custos

\begin{tabular}{c|cc}
\hline $\begin{array}{c}\text { Valor inicial da faixa } \\
(R \$)\end{array}$ & Valor final de faixa & Pes \\
\hline 0,00 & $400.000,00$ & 0 \\
$400.000,00$ & $800.000,00$ & 2 \\
$800.000,00$ & $1.200 .000,00$ & 3
\end{tabular}




\begin{tabular}{l|ll}
$1.200 .000,00$ & $1.600 .000,00$ & 4 \\
$1.600 .000,00$ & $2.000 .000,00$ & 5 \\
\hline
\end{tabular}

Tabela 4: Escala das urgências

\begin{tabular}{l|c}
\hline \multicolumn{1}{c|}{ Descrição da urgência } & $\begin{array}{c}\text { Pes } \\
\text { o }\end{array}$ \\
\hline $\begin{array}{l}\text { Não precisa alocar nada neste ano } \\
\text { Melhor alocar pelo menos 30\% do recurso neste }\end{array}$ & $\begin{array}{l}1 \\
\text { ano }\end{array}$ \\
$\begin{array}{l}\text { Melhor alocar pelo menos 30\% do recurso neste } \\
\text { ano }\end{array}$ & 5 \\
$\begin{array}{l}\text { Melhor alocar pelo menos 30\% do recurso neste } \\
\text { Me }\end{array}$ & 7 \\
\hline
\end{tabular}

Tabela 5: Escala dos Retornos

\begin{tabular}{l|c}
\hline \multicolumn{1}{c|}{ Descrição da } & Pes \\
urgência & $o$ \\
\hline Retorno muito baixo & 1 \\
Retorno baixo & 3 \\
Retorno médio & 5 \\
Retorno alto & 7 \\
Retorno muito alto & 9 \\
\hline
\end{tabular}

\subsection{Modelagem do Problema}

O problema estudado consiste na otimização da alocação recursos para os diversos projetos selecionados e priorizados conforme os pesos calculados pelo método AHP simplificado. Os projetos podem receber recursos fracionados, ou seja, que cubram ou não a totalidade do seu valor previsto, pois se considera que a parcela que faltar pode ser atendida em outra remessa de recursos. Tal diferenciação é considerada no critério "urgência" do método de definição de pesos.

As variáveis de decisão são $x_{i j}$, que representam os percentuais alocados em cada projeto $i$ da RM $j$. Os parâmetros utilizados são: $P_{i j}$ que representa o peso de cada projeto $i$ na $\mathrm{RM} j, C_{i j}$ representa o custo de cada projeto $i$ na $\mathrm{RM} j, R T$ representa o recurso total disponível para alocação, TS é o total solicitado pelas Regiões Militares, $F$ é um fator de redução que flexibiliza para menor o parâmetro de proporcionalidade RT/FS. O modelo proposto é apresentado a seguir.

$$
\operatorname{Max} Z=\sum_{i=1}^{n} \sum_{j=1}^{m} P_{i j} \cdot X_{i j}
$$

s.a.

$$
\begin{aligned}
& \sum_{i=1}^{n} \sum_{j=1}^{m}\left(C i \dot{i} i j \cdot x_{i j}\right) \leq R T i \\
& x_{i j} \cdot t_{i j} \leq P P, \forall i e j \\
& \left(\frac{R T}{T S} \cdot F\right) \cdot \sum_{i=1}^{n} C_{i j} \leq \sum_{i=1}^{n}\left(C i \dot{i} i j \cdot x_{i j}\right) ; \forall j i
\end{aligned}
$$




$$
\begin{aligned}
& x_{i j} \leq 1 ; \forall \text { ie } j \\
& x_{i j} \geq 0 ; \forall \text { ie } j
\end{aligned}
$$

A função objetivo (1) busca maximizar o recurso alocado, considerando o peso de cada projeto, onde $P_{i j}$ representa o peso de cada projeto. A restrição (2) garante que o valor total alocado nos projetos não exceda o recurso total disponível para alocação $R T$. As restrições (3) garantem que o tempo total de execução das obras não exceda o limite do tempo disponível para o planejamento $P P$. As restrições (4) garantem a proporcionalidade de distribuição nas Regiões Militares, para evitar que uma RM tenha um percentual de atendimento muito menor do que outra RM. Para fazer esta análise, calcula-se o percentual de atendimento geral (PA), sendo a razão entre o recurso total disponível e o recurso total solicitado dentre todas as RMs. O valor desta razão serve como um parâmetro para nortear qual em que ordem de grandeza deveria estar o percentual de atendimento para cada RM. Contudo, considerando que há outros fatores que interferem na otimização do recurso, como os pesos dos projetos e os tempos de obras, o percentual de atendimento geral deve ser flexibilizado por meio de um fator de redução (F), para que o problema tenha solução. As restrições (5) garantem que não será alocado mais do que $100 \%$ de cada projeto. As restrições (6) garantem que os percentuais não assumam valores negativos.

\section{ESTUDO DE CASO}

\subsection{A DiRETORIA DE OBRAS MILITARES}

A Diretoria de Obras Militares (DOM) é o órgão de apoio técnico-normativo do Departamento de Engenharia e Construção (DEC), incumbido de superintender, no âmbito do Exército, as atividades de construção, ampliação, reforma, adaptação, reparação, restauração, conservação, demolição e remoção de instalações, relacionadas à obras militares, e de controlar o material de sua gestão. A estrutura de obras militares do Exército é formada por um conjunto de macroprocessos finalísticos sob responsabilidade normativa e gerencial da DOM. Estes macroprocessos mapeiam todo o ciclo de vida de uma obra pública sob responsabilidade do Exército, indo desde a sua concepção até a demolição, cumprindo as fases de estudo de viabilidade, anteprojeto, projeto, planejamento, licitação, contratação, acompanhamento, fiscalização, controle e conclusão; e após a entrega da obra, a nova edificação entra no ciclo de manutenção.

A DOM é a gestora do macroprocesso de obras militares e é responsável pela escolha e priorização dos projetos que serão desenvolvidos e licitados. Após o processo licitatório, esta diretoria distribui os recursos financeiros disponíveis dentre as variadas obras. Para tal ela adota critérios de prioridade entre as obras já iniciadas e as que vão iniciar e aloca os recursos tentando aproveitar ao máximo a disponibilidade financeira. Este artigo busca fornecer uma ferramenta de apoio à decisão para priorização das obras que receberão recurso e na definição dos valores alocados em cada uma delas.

\subsection{As Regiões Militares}

O Exército Brasileiro possui 650 organizações militares (OM) que ocupam patrimônio jurisdicionado e realizam solicitações de manutenção e construção. Estas OM estão distribuídas em 12 comandos de regiões militares (RM). Os 4 comandos de grupamentos de engenharia (Gpt E) realizam a priorização das necessidades regionais. As 9 Comissões Regionais de Obras (CRO) e os 3 Serviços Regionais de Obras (SRO) são responsáveis pela execução de obras em sua respectiva Região Militar. 


\subsection{O PROCESSO DECISÓRIO}

O processo de custeio de uma obra tem diversas etapas desde concepção de objetivos estratégicos do Exército Brasileiro, até processos técnicos como confecção de projetos e licitações. O alvo deste trabalho está na parte final do processo onde o projeto está pronto, licitado e a obra contratada. Neste momento o decisor deverá definir quanto vai disponibilizar de recurso para iniciar (ou continuar) a execução de uma determinada obra. Para o desenvolvimento deste trabalho serão adotadas as considerações:

- As obras poderão receber percentuais fracionários dos seus orçamentos originais (custo da obra).

- O valor alocado para cada obra será aplicado ao longo de um período de planejamento pré-definido. Por exemplo, se o período do planejamento é 12 meses, o recurso alocado em uma determinada obra será aplicado ao longo de 12 meses, e após este período uma nova distribuição de recursos será feita.

- Cada região militar apresentará os valores necessários para iniciar (ou continuar) cada uma de suas obras. O somatório destes valores dentre todas as RM será o total solicitado. Calcula-se então o percentual de atendimento geral pela relação entre o recurso total disponível e o total solicitado. Cada RM deverá ser atendida considerando um percentual mínimo de atendimento, a ser calculado tomando como referência o percentual de atendimento geral reduzido por um fator de redução.

\subsection{DAdOS DO ESTUdO}

Para este estudo de caso, selecionou-se o portfólio de projetos do Sistema de Saúde do Exército Brasileiro. Este portfólio é constituído por 38 projetos cujos custos estão organizados na Tabela 3 e distribuídos em 7 das 12 RMs. Devido ao carácter sensível das informações dos projetos, o teor dos projetos não será descrito neste artigo, apenas seus custos orçados.

Tabela 6: Custos dos projetos

\begin{tabular}{|c|c|c|c|c|}
\hline $\begin{array}{l}\text { Custos } \\
\text { dos } \\
\text { projetos }\end{array}$ & $1^{a} R M$ & $2^{a} R M$ & $3^{a} R M$ & $4^{a} R M$ \\
\hline P1 & $\mathrm{R} \$ 2.000 .000,00$ & $\mathrm{R} \$ 200.000,00$ & $\mathrm{R} \$ 1.446 .232,67$ & $\mathrm{R} \$ 642.151,60$ \\
\hline P2 & $\mathrm{R} \$ 1.000 .000,00$ & $\mathrm{R} \$ 150.000,00$ & $\mathrm{R} \$ 750.000,00$ & \\
\hline P3 & $\mathrm{R} \$ 500.000,00$ & $\mathrm{R} \$ 90.000,00$ & $\mathrm{R} \$ 66.699,75$ & \\
\hline P4 & $\mathrm{R} \$ 445.000,00$ & $\mathrm{R} \$ 350.000,00$ & $\mathrm{R} \$ 500.000,00$ & \\
\hline P5 & $\mathrm{R} \$ 315.000,00$ & & $\mathrm{R} \$ 500.000,00$ & \\
\hline P6 & $\mathrm{R} \$ 405.840,00$ & & $\mathrm{R} \$ 800.000,00$ & \\
\hline P7 & $\mathrm{R} \$ 95.000,00$ & & $\mathrm{R} \$ 109.696,25$ & \\
\hline P8 & $\mathrm{R} \$ 100.000,00$ & & $\mathrm{R} \$ 700.000,00$ & \\
\hline P9 & $\mathrm{R} \$ 455.000,00$ & & $\mathrm{R} \$ 240.000,00$ & \\
\hline P10 & $\mathrm{R} \$ 200.000,00$ & & $\mathrm{R} \$ 548.000,00$ & \\
\hline P11 & $\mathrm{R} \$ 175.000,00$ & & $\mathrm{R} \$ 23.026,80$ & \\
\hline P12 & & & $\mathrm{R} \$ 15.359,50$ & \\
\hline Total & $\mathrm{R} \$ 5.690 .840,00$ & $\mathrm{R} \$ 790.000,00$ & $\mathrm{R} \$ 5.699 .014,97$ & $\mathrm{R} \$ 642.151,60$ \\
\hline $\begin{array}{l}\text { Custos } \\
\text { dos } \\
\text { projetos }\end{array}$ & $6^{a} R M$ & $7^{a} R M$ & $9^{a} R M$ & \\
\hline P1 & $\mathrm{R} \$ 50.000,00$ & $\mathrm{R} \$ 156.670,00$ & $\mathrm{R} \$ 693.000,00$ & \\
\hline
\end{tabular}




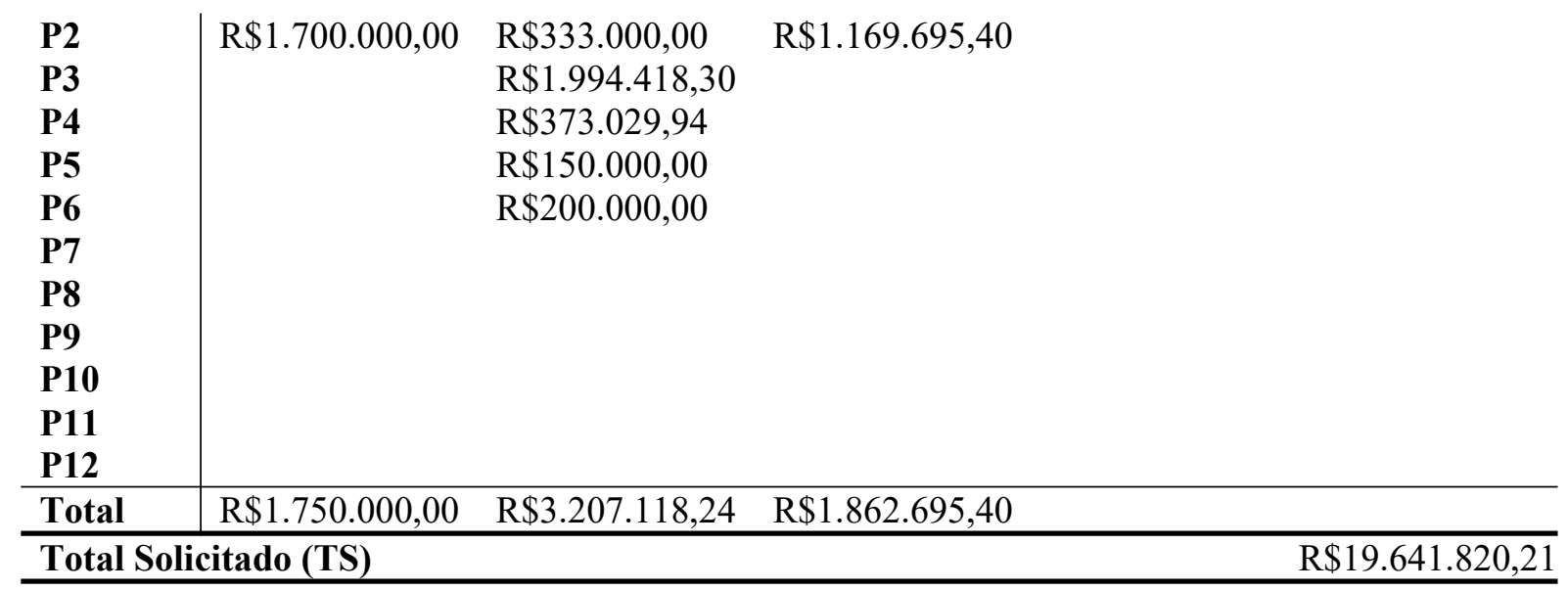

Após a execução do método SPC, chega-se aos pesos dos projetos, conforme Tabela 7.

Tabela 7: Pesos dos projetos

\begin{tabular}{|c|c|c|c|c|c|c|c|}
\hline $\begin{array}{l}\text { Pesos } \\
\text { dos } \\
\text { projetos }\end{array}$ & $\begin{array}{r}1^{a} \\
R M\end{array}$ & $\begin{array}{r}2^{a} \\
R M\end{array}$ & $\begin{array}{r}3^{a} \\
R M\end{array}$ & $\begin{array}{r}4^{a} \\
R M\end{array}$ & $\begin{array}{r}\boldsymbol{G}^{a} \\
R M\end{array}$ & $\begin{array}{r}7^{a} \\
R M\end{array}$ & $\begin{array}{r}9^{a} \\
R M\end{array}$ \\
\hline $\mathbf{P 1}$ & $\begin{array}{r}0,0 \\
5\end{array}$ & 0,03 & 0,06 & $\begin{array}{r}0,0 \\
4\end{array}$ & 0,08 & $\begin{array}{r}0,0 \\
4\end{array}$ & 0,04 \\
\hline P2 & $\begin{array}{r}0,0 \\
4\end{array}$ & 0,01 & 0,05 & & 0,07 & $\begin{array}{r}0,0 \\
4\end{array}$ & 0,05 \\
\hline P3 & $\begin{array}{r}0,0 \\
5\end{array}$ & 0,01 & 0,05 & & & $\begin{array}{r}0,0 \\
5\end{array}$ & \\
\hline P4 & $\begin{array}{r}0,0 \\
5\end{array}$ & 0,01 & 0,06 & & & $\begin{array}{r}0,0 \\
5\end{array}$ & \\
\hline P5 & $\begin{array}{r}0,0 \\
5\end{array}$ & & 0,06 & & & $\begin{array}{r}0,0 \\
3\end{array}$ & \\
\hline P6 & $\begin{array}{r}0,0 \\
4\end{array}$ & & 0,05 & & & $\begin{array}{r}0,0 \\
3\end{array}$ & \\
\hline P7 & $\begin{array}{r}0,0 \\
2\end{array}$ & & 0,04 & & & & \\
\hline P8 & $\begin{array}{r}0,0 \\
3\end{array}$ & & 0,05 & & & & \\
\hline P9 & $\begin{array}{r}0,0 \\
4\end{array}$ & & 0,04 & & & & \\
\hline P10 & $\begin{array}{r}0,0 \\
2\end{array}$ & & 0,05 & & & & \\
\hline $\begin{array}{l}\text { P11 } \\
\text { P12 }\end{array}$ & $\begin{array}{r}0,0 \\
1\end{array}$ & & $\begin{array}{l}0,03 \\
0,03\end{array}$ & & & & \\
\hline
\end{tabular}

Uma vez calculados os pesos dos projetos, a próxima etapa é a utilização das ferramentas de programação linear para otimização da alocação de recursos a partir da função objetivo e restrições. Para isso, o autor criou dados fictícios de tempo previsto para cada projeto, de recurso total disponível para o portfólio e o Fator de Redução de proporcionalidade entre as RM. A partir destes dados, foi calculado o parâmetro percentual de atendimento (PA) e calculados os intervalos de alocação de recurso para cada RM.

Tabela 8: Tempos das obras

\begin{tabular}{l|lllllll}
\hline Tempos & $1^{a}$ & $2^{a}$ & $3^{a}$ & $4^{a}$ & $6^{a}$ & $7^{a}$ & $9^{a}$ \\
\hline
\end{tabular}




\begin{tabular}{l|rrrrrrr}
\hline $\begin{array}{l}\text { de Obra } \\
\text { (meses) }\end{array}$ & $\boldsymbol{R}$ & $\boldsymbol{R}$ & $\boldsymbol{R}$ & $\boldsymbol{R}$ & $\boldsymbol{R}$ & $\boldsymbol{R}$ & $\boldsymbol{R}$ \\
\hline P1 & 24 & 10 & 24 & 18 & 6 & 6 & 13 \\
P2 & 18 & 8 & 17 & & 20 & 10 & 24 \\
P3 & 14 & 6 & 9 & & & 24 & \\
P4 & 15 & 14 & 13 & & & 13 & \\
P5 & 13 & & 14 & & & 14 & \\
P6 & 14 & & 18 & & & 12 & \\
P7 & 13 & & 12 & & & & \\
P8 & 13 & & 16 & & & & \\
P9 & 15 & & 8 & & & & \\
P10 & 14 & & 10 & & & & \\
P11 & 18 & & 6 & & & & \\
P12 & & & 6 & & & & \\
\hline
\end{tabular}

Tabela 9: Dados do estudo

\begin{tabular}{l|r}
\hline \multicolumn{1}{c|}{ Recurso total disponível } & $\mathrm{R} \$ 17.000 .000,00$ \\
RT) & \\
Recurso total solicitado (TS) & $\mathrm{R} \$ 19.641 .820,21$ \\
$\mathrm{PA}$ & 0,87 \\
$\mathrm{~F}$ & 0,70 \\
PA*F & 0,61 \\
Período de planejamento & 12 meses \\
\hline
\end{tabular}

Utilizando o modelo matemático e os dados de entrada apresentados, realizou-se a modelagem e o carregamento dos dados na versão acadêmica do software de modelagem matemática AIMMS.

\subsection{Resultados}

Ao inserir os dados no AIMMS com Fator de Redução de 0,9, o programa retornou que a otimização era impossível. Isso se deve a influência das outras restrições do problema. Diminui-se gradualmente o valor de $\mathrm{F}$ até que o foi obtida solução para F=0,7.

Tabela 10: Resultados - Valores de $x_{i j}$

\begin{tabular}{l|rrrrrrr}
\hline Valore & $\mathbf{1}^{\boldsymbol{a}}$ & $\mathbf{2}^{\boldsymbol{a}}$ & $\mathbf{3}^{\boldsymbol{a}}$ & $\mathbf{4}^{\boldsymbol{a}}$ & $\boldsymbol{6}^{\boldsymbol{a}}$ & $\mathbf{7}^{\boldsymbol{a}}$ & $\mathbf{9}^{\boldsymbol{a}}$ \\
s de $\boldsymbol{x}_{i j}$ & $\boldsymbol{R M}$ & $\boldsymbol{R M}$ & $\boldsymbol{R M}$ & $\boldsymbol{R M}$ & $\boldsymbol{R M}$ & $\boldsymbol{R M}$ & $\boldsymbol{R M}$ \\
\hline P1 & 0,5 & 1 & 0,5 & 0,66 & 1 & 1 & 0,92 \\
P2 & 0,6 & 1 & 0,7 & & 0,6 & 1 & 0,50 \\
& 7 & & 0 & 0 & & \\
P3 & 0,8 & 1 & 1 & & & 0,5 & \\
& 5 & & & & & 0 & \\
P4 & 0,8 & 0,85 & 0,9 & & & 0,9 & \\
& 0 & & 2 & & & 3 & \\
P5 & 0,9 & & 0,8 & & & 0,8 & \\
& 2 & & 5 & & & 5 & \\
P6 & 0,8 & & 0,6 & & & 1 & \\
& 5 & & 6 & & & & \\
P7 & 0,9 & & 1 & & & & \\
& 3 & & & & & & \\
P8 & 0,9 & & 0,7 & & & & \\
& 2 & & 5 & & & &
\end{tabular}




\section{SPOLM2O19}

XIX SIMPÓSIO DE PESQUISA OPERACIONAL E LOGÍSTICA DA MARINHA RIO DE JANEIRO, RJ, BRASIL - 06 A 08 DE NOVEMBRO DE 2019

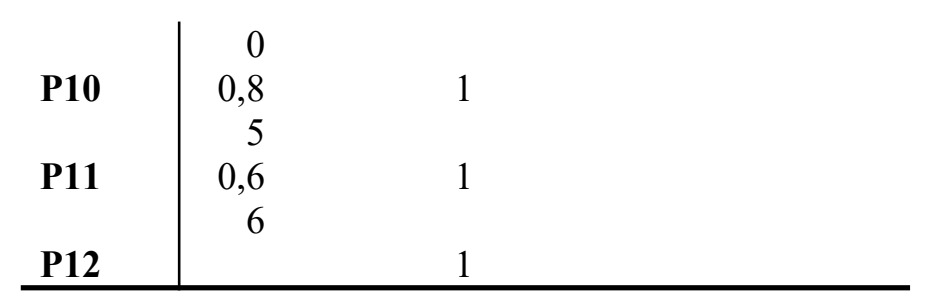

Considerando os valores solicitados para cada obra em cada Região Militar, a tabela 10 mostra qual o percentual otimizado de cada valor de obra que deverá ser disponibilizado, de forma a atender às restrições impostas e minimizar o custo total. Ou seja, tomando como exemplo o projeto $\mathrm{P} 1$ da $1^{\mathrm{a}} \mathrm{RM}$, no período de planejamento inicial de 12 meses, devem ser disponibilizados 50\% do valor solicitado, ou seja, R\$1.000.000,00.

\subsection{CONCLUSÃo}

A otimização da aplicação dos recursos é preocupação fundamental para qualquer instituição. Contudo, quando se trata de órgãos e instituições públicas, a otimização adquire obrigação do gestor. O método Structured Pair-wise Comparison é uma forma prática de comparar critérios e valorar alternativas sob o ponto de vista de cada critério. Tal método colabora para a atribuição de pesos aos projetos substituindo o caráter intuitivo ainda usado por alguns gestores.

Uma vez determinado o modelo matemático, o software utilizado se apresenta como excelente ferramenta para cálculo da otimização, contudo, deve-se considerar que o gestor deverá entender a influência das variáveis no modelo. Isso decorre do fato de que algumas combinações de restrições e valores de dados podem tornar o problema sem solução, fazendo com o que o gestor saiba ajustar seus dados flexibilizando o modelo, a exemplo do ocorrido com o valor de F, no estudo de caso.

Observa-se que os pesos dos projetos influenciam consideravelmente no resultado da otimização, assim como a restrição r3, que trata do período de planejamento. Os valores encontrados para $x_{i j}$ refletem o percentual do custo de cada obra que deverá ser alocado no período de planejamento, ou seja, em 12 meses. Ao aplicar estes percentuais, a DOM utilizará o recurso disponível de forma otimizada, considerando os custos, urgências e retornos de cada obra, assim como as restrições específicas do seu portfólio.

\section{REFERÊNCIAS BIBLIOGRÁFICAS}

[1] CAMPOS, V. F. TQC - Controle da Qualidade Total: no estilo japonês. 9a. ed. Belo Horizonte, MG: Editora Falconi, 2014.

[2] PMI. The Standard for Portfolio Management. Pensilvância, EUA: Project Management Institute, Inc, 2013.

[3] PROJECT MANAGEMENT INSTITUTE (PMI). A guide to the project management body of knowledge. PMBOK Guide, USA, 2006.

[4] PMBOK. PMI. Um Guia do Conhecimento em gerenciamento de projetos [s.l: s.n.]. v. 1

[5] BRASIL. Ministério do Planejamento, Orçamento e Gestão. Secretaria de Logística e Tecnologia da Informação. Metodologia de Gerenciamento de Projetos do SISP /Ministério do Planejamento, Orçamento e Gestão, Secretaria de Logística e Tecnologia da Informação. Brasília: MP, 2011. 
[6] Disponível em: <http://www.sisp.gov.br/mgpsisp/wiki/Apresentacao>. Acesso em: 11 maio 2019.

[7] Archer, N. ., \& Ghasemzadeh, F. (1999). An integrated framework for project portfolio selection. International Journal of Project Management, 17(4), 207-216.

[8] CATELLI, A.; et al. Sistema de Gestão Econômica - GECON. In: CATELLI, A. (Coord.). Controladoria: uma abordagem da gestão econômica - GECON. São Paulo: Atlas, 2001. p.285-307.

[9] LIESIÖ, J.; MILD, P.; SALO, A. Robust portfolio modeling with incomplete cost information and project interdependencies. European Journal of Operational Research, v. 190, n. 3, p. 679-695, 2008.

[10] GOMES L.F.A.M. Teoria da Decisão. São Paulo: Editora Thompson, 2007. 116p. (Coleção Debates em Administração). Bibliografia: p. 1-20. ISBN 85-221-0529-4.

[11] SAATY T. L. Fundamentals of Decision Making and Priority Theory with the Analytic Hierarchy Process. Pittsburgh: RWS publications, 2006. 478p., 21cm. (Vol VI of the AHP series). Bibliografia: p.8-92 ISBN 0-9620317-6-3.

[12] GOMES, L.F.A.M.; ARAYA, M.C.G.; CARIGNANO, C. Tomada de decisões em cenários complexos. São Paulo: Thompson, 2004.

[13] SILVA, L. M. ; SILVA R. da. Planejamento estratégico de uma experiência pedagógica inovadora. Revista Minerva, v. 6, p. 99-106, 2009.

[14] OLIVEIRA, F. F.; FERREIRA, R. J. P. Análise das Contribuições da Pesquisa Operacional para as Decisões de Layout em Gestão da Produção: Uma Revisão da LiteraturaXXXI Encontro Nacional de Engenharia de Produção. Anais...Belo Horizonte, MG: 2011

[15] MOREIRA, D. A. Pesquisa Operacional: Curso Introdutório. São Paulo, SP: Thompson Learning, 2010.

[16] GOLDBARG, M. C.; LUNA, H. P. L. Otimização combinatória e programação linear: modelos e algoritmos. [s.l: s.n.]. 